\title{
The Review on the Function of Intestinal Flora and the Regulatory Effects of Probiotics on the Intestinal Health of Rabbits
}

\author{
Hong-ju CHEN ${ }^{a}$, Wan-ying YANG ${ }^{b}$ and Chun-yang WANG ${ }^{c,}$
}

Shandong Provincial Key Laboratory of Animal Biotechnology and Disease Control and Prevention, Shandong Agricultural University, 61 Daizong Street, Taian City, Shandong Province, 271018, China

ahjchen72@sdau.edu.cn, b1070329051@qq.com, 'wcy@sdau.edu.cn

${ }^{*}$ Corresponding author

Keywords: Intestine, Microflora, Probiotic, Rabbit

\begin{abstract}
Rabbits are both monogastric and herbivore animals, with special digestive and physiological characteristics. The establishment of healthy, stable and diverse digestive tract microflora is of great significance for rabbits to resist intestinal diseases.Probiotics have been defined as living bacteriawhich modulate the gut microflora and improvethe health of the host beyond their inherent basicnutrition. The purpose of this paper is to summarize the role of intestinal microbes and the effects of probiotics on their intestinal microbes.
\end{abstract}

\section{Introduction}

Rabbits are both monogastric animals, but also herbivores, with special digestive and physiological characteristics. The rabbit farming is an important emerging enterprise in many countries of the world. Studies have found that in a rabbit field which has a mortality rate of $24 \%, 75 \%$ of deaths were caused by diarrhea. A high incidence of digestive diseases in rabbits often related to distortions in microflora composition[1,2]. Any imbalance of microflora can result in alteration of $\mathrm{pH}$, dysbiosis and proliferation of pathogens, with detrimental effects on the animal's health[3].The establishment of healthy, stable and diverse digestive tract microflora is of great significance for rabbits to resist intestinal diseases.

Antibiotics have been widely used in animal production to resistant pathogenic and protect the health of gut. Unfortunately, the long term and extensive use of antibiotics has led to the appearance of worrying bacterial drug resistance and stressed the problem of food residues, which may cause problems for human health[4]. The European Union Commission banned the use of antibiotics as a growth promoter in animal diets, and consumers as well as breeders require alternative breeding strategies that improve the health of animals without using in-feed antibiotics. This situation stimulated research to explore alternatives to antibiotic growth promotants. These non-antibiotic compounds with bacteriostatic or bactericidal activity are probiotics, prebiotics, bacteriocins and organic acids, etc[5,6].

Probiotics are direct-fed microbial feed supplements which modulate the gut microflora by successfully competing with pathogens through a competitive exclusion process[7]. Available probiotics include the Lactic acid bacteria, Enterococci, Bifidobacteria, Yeasts and so on. They are extensively used in the medical and veterinary fields to treat and reduce the symptoms of many gastrointestinal diseases and as a preventive approach to maintain a healthy gut flora[8-10]. Previous studies 
on intestine biodiversity of rabbits performed using classical culture-based techniques and recently, by molecular techniques.

The aim of this review is to focus on the relation between probiotics and gut health of rabbits.

\section{The Function of Intestinal Flora in Rabbits}

\section{Promote Feed Digestion and Nutrient Utilization}

The nutrients of the intestinal flora come from the host's digestive tract, when the microorganisms obtain nutrients, the digestive enzymes secreted are beneficial to the decomposition of feed, which can promote host's utilization of nutrients. Rabbits' cecum microorganisms can ferment foregut undigested cellulose and other nutrients, volatile fatty acids, ammonia nitrogen and vitamins and other nutrients. Volatile fatty acid is rapidly absorbed in the intestine, then can provide $40 \%$ maintaining energy to adult rabbits, in which butyric acid is a direct source of energy to hindgut, while acetic acid creates cholesterol and fat metabolism in the liver in metabolism[11]. In addition, the microbes in the cecum can also use ammonia nitrogen to the synthesis of bacterial proteins, these proteins are used twice by rabbits in the form of soft feces [12]. Normal intestinal flora is beneficial to host's digestion and metabolism of nutrients, thereby promoting the growth and development of rabbits. Feed provides nutrients and energy for animal's intestinal ecosystem, which is the main factor affecting adult animal's intestinal microbial balance. The bacterial structure affects degradation of feed and therefore affects the physicochemical parameters of intestinal $\mathrm{PH}$, metabolite concentration and redox potential. On the contrary, the dietary composition also affects the balance of intestinal flora[13]. The digestive physiology of rabbits is largely based on its caecal microbial population, characterized by the presence of an abundant microflora.

\section{Intestinal Barrier Function}

Normal intestinal flora maintains the stability of the intestinal habitat, and inhibit the colonization of harmful bacteria in many ways, and provide a protective barrier in order to resist the invasion of exogenous pathogens to prevent the occurrence of intestinal inflammation to ensure intestinal integrity. First, normal flora competitively adhere to the intestinal epithelial cells through the mass effect to prevent the adhesion of pathogens, so that retention time of foreign microorganisms in the intestinal is shortened and be discharged rapidly with chyme. At the same time, normal bacteria can also secrete antibiotics and other antibacterial substances to inhibit the propagation of pathogens. In addition, normal intestinal microflora can competitively inhibit the survival and proliferation of harmful flora by competing for nutrients in the intestinal habitat $[14,15]$.

\section{Promote the Development of Intestinal Tract}

Small intestine is the main site of digestion and absorption of animal nutrients, villus length and crypt depth reflect the state of digestion and absorption of the small intestine. Balance of intestinal microflora helps to improve the height of villus and reduce the depth of crypts, and promote intestinal development. Nishio[16]found that addition of lactic acid bacteria and chicken cecal contents to sterile diets in sterile chickens significantly increased duodenal and jejunum villus height / crypt depth. Slezak et al.[17]found that the length of small intestine in sterilized mice was significantly smaller than that in normal ones. In addition, the division, differentiation, 
and renewal of intestinal mucosal cells require appropriate immune stimulation, the normal microflora structure of the intestine produces a corresponding stimulating effect to ensure intestinal mucosal integrity[18].

\section{Stimulate Immune Organ Development and Regulate Host Immune Function}

The normal flora in the cecum of rabbits is necessary for their development and maturation. Earthworm process is a specific immune organ of rabbits, which contains hundreds of lymphoid follicles, follicular-related intracellular $\mathrm{M}$ cells migrate by cecal microbials' stimulation to the intestinal mucosa to resist intestinal infection, maintain intestinal health[19,20]. There exist widespread lymphocytes in Intestinal mucosa lamina, which can turn into plasma cells when stimulated by the normal flora, resulting in $\operatorname{IgA}$. When IgA turns secreted IgA through the mucosal epithelium to the mucosal surface, it has a strong resistance to infection [21,14]. Intestinal flora can also significantly activate macrophage's activity, promote secretion of cytokine mediators, improve host resistance to disease[22,23]. Rhee et al.[24]found that inoculating B.subtilis and B.fragilis at the same time to sterile rabbit can promote B cell's proliferation and immunoglobulin's secretion. Therefore, the regulation of normal microbial balance in the intestine of rabbits has important significance for improving feed utilization, promoting intestinal development and mucosal maturation, reducing invasion of exogenous pathogens and improving production performance and immunity.

Since the gut microflora is beneficial to the host, hosts have evolved intestine-specific immune systems to co-exist with themicroflora. On the other hand, the intestinalmicroflora actively regulates the host's immune system, and recent studies have revealed that specific commensal bacterial species induce the accumulation of specific immune cell populations[16]. The immune cells induced by the gutmicroflora likely contribute to intestinal homeostasis and influence systemic immunity in the host.

\section{Effects of Probiotics on Intestinal Microflora in Rabbits}

Probiotics are live microbial cultures of non-pathogenic bacteria or yeast species that help to equilibrate the intestinal microflora. Previous studies with rabbits indicated probiotics supplementation improves growth rate, enhances efficiency of feed conversion in rabbits, and also influences the intestinal microflora through the action of beneficial microbes[8,25]. The stable or improved intestinal environment and gut health directly influence the health status and growth performance of animals due to better nutrient absorption in the gut. The beneficial bacteria can also promote the propagation of beneficial microorganisms by reducing the oxygen in digestive tract or reducing the intestinal $\mathrm{pH}$, and improve the intestinal tract habitat to antagonize the proliferation of pathogenic bacteria, thereby optimizing the intestinal flora structur[26].The Growth performance, and immune function of probiotics on the rabbit have been described in several reviews[27,28].

Few studies have been published on the use of probiotics in the management of gastrointestinal diseases and little is known about their effects on the caecal and faecal microbial community. Despite this, probiotics are routinely administered, along with conventional treatments, to alleviate the clinical signs of gastrointestinal disease in pet rabbits, and as a prophylactic to minimise the potential adverse effects of antibiotic usage on the gastrointestinalflora. So, the present review focuses on the effection of probiotics on gut microflora of rabbits. 
Copeland et al.[10]showed that the addition of probiotics to diets could reduce the number of harmful microbes such as E. coli and Salmonella. Studies found that exogenous addition of probiotics could promote intestinal mucosal regeneration and integrity.

Seyidoglu and Peker[26]concluded that low $(2 \mathrm{~g} / \mathrm{kg})$ or high doses $(4 \mathrm{~g} / \mathrm{kg})$ may be used for intestinal health of adult rabbits, for example: the total thickness of the mucosa, villus heights, crypt depths and gland depths were increased significantly. This condition has been resulted indecreasethe surface area for nutrient absorption.

Yakabe et al.[29]suggested Lactobacillus brevis KB290 improves gut health and stimulates immune function., Which would be safe for pregnant females and their offspring.Simonova et al.[30]conclude that administration of Enterococcus faecium CCM7420 $\left(5.0 \times 10^{8} \mathrm{CFU} / \mathrm{animal} / \mathrm{day}\right.$ in their drinking water $)$ strain to rabbits may improve weight gain due to better utilization of feed and larger absorption surface in the gut and also may positively influence the health status via enhancing the gut health in rabbits.Live yeast Saccharomyces cerevisiae NCYC Sc 47 was added to the diet of weaning rabbits (35d old) to analyse the effects on the caecal ecosystem (biotope and bacterial community), digestion and health status. The structure of the caecal bacterial community was not modified after $11 \mathrm{~d}$ of yeast presence in the caecum, while the bacterial diversity tended to be higher[31].Campos-Morales et $a l .[25]$ conclude that yeast supplementation in the volcano rabbit negatively affects digestion and mortality in captivity.Benato et al.[9]investigate the effects of probiotics on faecalmicroflora shown that oral administration of probiotic E. faecium NCIMB 30183 to pet rabbits was associated with an increased level of faecal E. faecium after 2 weeks of dietary supplementation, but did not influence the levels of $C$. spiroforme, Bacteroides species and $F$. succinogenes.Oso et al.[5]reported that the diet of adult rabbits containing probiotics (Prediococcus acidilactis, Bacillus cereus) enhance the caecal lactobacillus but lower the coliform counts. Meanwhile, the Clostridium count was not affected. Wang et al.[32]conclued that Lactobacillus isolates LB1 and L3 can alter the gut microbiota to the direction of more abundance and diversity, particularly in the cecum,by pyrosequencing-based analysis .Simonova et al.[30]performed a similar study whereby E. faecium CCM7420 was administrated as a probiotic in rabbits for 21 and 42 days and reported that the probiotic increased the counts of Enterococci but was unable to modulate the levels of other faecal bacteria such as Staphylococci and Staphylococcus aureus.

In summary, probiotics can enhance the activity of digestive enzymes, promote intestinal digestion and absorption of nutrients, so as to improve the growth performance of rabbits and maintain intestinal health by promoting the integrity of the intestinal mucosa and the growth of beneficial bacteria.

\section{Acknowledgement:}

This research was financially supported by the Funds of Shandong "Double Tops" Program

\section{Reference}

[1]Combes,S., Michelland,R. J.,Monteils, V.,et,al. Postnatal development of the rabbit caecal microbiota composition and activity. FEMS Microbiol Ecol., 77 (2011): 680689. 
[2]Bauerl,C., Collado,M.C., et,al.. Changes in cecal microbiota and mucosal gene expression revealed new aspects of epizootic rabbit enteropathy. PLoS One., 9 (2014):105-107.

[3]Michelland,R.J.,Combes,S.,Monteils,V., et,al. Molecular analysis of the bacterial community in digestive tract of rabbit. Anaerobe., 16 (2010):61-65.

[4]Wang, C.Y., Li,F.C.,Zhu,Y.L., et,al.Impact of probiotics on intestinal microbial community diversity of growing Rex Rabbits. 10 th World Rabbit Congress:( 2012) 635-639.

[5]Oso,A.O.,Idowu,O.M.O., et,al.. Growth performance, apparent nutrient digestibility, caecal fermentation, ileal morphology and caecal microflora of growing rabbits fed diets containing probiotics and prebiotics. Livestock Science., 157(2013):184-190.

[6]Olorunsola, R., Akinduti, P.,Oso, A., et,al.. Effect of Dietary Supplementation with Probiotics and Prebiotics on Haematological Indices, Serum Chemistry and Gut Salmonella Count of Broilers Sourced from Salmonella- inf- ected Hatcheries in South-west Zone of Nigeria. American Journal of Experimental Agriculture., 10 (2016):1-13.

[7]Gerritsen, J., Smidt, H.,Rijkers, G. T.,de Vos, W. M.. Intestinal microbiota in human health and disease: the impact of probiotics. Genes Nutr., 6 (2011):209-240.

[8]Abdel-Aziz,N.A., El-Adawy,M. , et,al.. Effects of exogenous enzymes, Lactobacillus acidophilus or their combination on feed performance response and carcass characteristics of rabbits fed sugarcane bagasse. Journal of Integrative Agriculture., 14(2015):544-549.

[9]Benato, L., Hastie,P., Shaughnessy, et,al. Effects of probiotic Enterococcus faecium and Saccharomyces cerevisiae on the faecal microflora of pet rabbits. Journal of Small Animal Practice., 55(2014):442-446.

[10]Copeland, D. R., McVay, M. R , et,al.. Probiotic fortified diet reduces bacterial colonization and translocation in a long-term neonatal rabbit model. Journal of Pediatric Surgery ., 44(2009):1061-1064.

[11]Gidenne, T.. Caeco-colic digestion in the growing rabbit: impact of nutritional factors and related disturbances. Livestock Production Science., 51 (1997):73-88.

[12]Kohl, K. D., Weiss, R.B., Dearing,M.D.. Diversity and novelty of the gut microbial community of an herbivorous rodent (Neotoma bryanti). Symbiosis., 54 (2011):47-54.

[13]Zhu, Y.L., Wang, C.Y., Li, F.C.. Impact of dietary fiber/starch ratio in shaping caecal microbiota in rabbits. Can. J. Microbiol., 61(2015): 771-784.

[14]Goto, Y. and Kiyono, H.. Epithelial barrier: an interface for the cross-communication between gut flora and immune system. Immunol Rev.,245(2012):147-163.

[15]Tremaroli, V.and Backhed, F. Functional interactions between the gut microbiota and host metabolism. Nature., 489 (2012):242-249. 
[16]Nishio, J.and Honda, K.. Immunoregulation by the gut microbiota. Cell Mol Life Sci., 69 (2012):3635-3650.

[17]Slezak, K., Krupova, Z.,Rabot, S., et,al. Association of germ-free mice with a simplified human intestinal microbiota results in a shortened intestine. Gut Microbes., 5 (2014):176-182.

[18]Garrett, W. S., Gordon, J. I.,Glimcher, L. H. Homeostasis and inflammation in the intestine. Cell., 140 (2010.): 859-870.

[19]Guarner, F. and Malagelada, J. R.. Gut flora in health and disease. The Lancet., 361(2003):512-519.

[20]Fortun-Lamothe, L. and Boullier, S.. A review on the interactions between gut microflora and digestive mucosal immunity. Possible ways to improve the health of rabbits. Livestock Science., 107(2007): 1-18.

[21]Pils, M.C., Bleich, A.,Prinz, I., et,al. Commensal gut flora reduces susceptibility to experimentally induced colitis via T-cell-derived interleukin-10. Inflamm Bowel Dis., 17 (2011): 2038-2046.

[22]Umesaki, Y. and Setoyama,H.. Structure of the intestinal flora responsible for development of the gut immune system in a rodent model. Microbesand Infection., 2(2000):1343-1351.

[23]Heller, F. and Duchmann, R.D.. Intestinal flora and mucosal immune responses. Int J Med Microbiol., 293 (2003): 77-86.

[24]Rhee, K.J., Sethupathi, P.,Driks, et,al.. Role of Commensal Bacteria in Development of Gut-Associated Lymphoid Tissues and Preimmune Antibody Repertoire. The Journal of Immunology., 172 (2004):1118-1124.

[25]Campos-Morales, R., Mendoza,G.D. , Ojeda,J. et,al. The effect of Saccharomyces cerevisiae on digestion and mortality in the volcano rabbit (Romerolagus diazi). Journal of Integrative Agriculture., 14 (2015):520-525.

[26]Seyidoglu,N.and Peker,S. Effects of different doses of probiotic yeast Saccharomyces cerevisiae on the duodenal mucosa in rabbits. Indian Journal of Animal Research., 49 (2015):602-606.

[27]Kalma, R. P., Patel, V. K. , Joshia, A. , et,al. Probiotics supplemention in rabbit: a review. International Journal of Agriculture Sciences., 8 (2016):2811-2815.

[28]Heselmans, M., Reid, G. , Akkermans,L.M.A. et,al. Gut Flora in Health and Disease: Potential Role of Probiotics. Curr. Issues Intestinal Microbiol., 6(2004):1-8.

[29]Yakabe,T., Takashima,H., Kuwagata,M., et,al. Teratogenicity and maternal effects of Lactobacillus brevis KB290 in rats and rabbits. Food and Chemical Toxicology., 49(2011):722-726.

[30]Simonová, M.P., Lauková, A.,Žitňan, R.,Chrastinová, L. Effect of rabbit-origin enterocin-producing probiotic strain Enterococcus faecium CCM7420 application on growth performance and gut morphometry in rabbits. Czech Journal of Animal Science., 60 (2015):509-512. 
[31]Kimsé, M., Bayourthe, C.,Monteils, V., et,al. Live yeast stability in rabbit digestive tract: Consequences on the caecal ecosystem, digestion, growth and digestive health. Animal Feed Science and Technology., 173(2012):235-243.

[32]Wang, C.Y., Zhu,Y.L., Li, F.C., Huang, L.B.. The Effect of Lactobacillus isolates on growth performance, immune response, intestinal bacterial community composition of growing Rex Rabbits. J Anim Physiol Anim Nutr (Berl)( 2017): 1-13. 